\title{
Challenging a Preconception: Optoacoustic Spectrum Differs from the Optical Absorption Spectrum of Proteins and Dyes for Molecular Imaging
}

Juan Pablo Fuenzalida Werner, ${ }^{\dagger, ¥}$ Yuanhui Huang, ${ }^{\dagger, \S, ¥}$ Kanuj Mishra, ${ }^{\dagger, \S}$ Robert Janowski, ${ }^{\times}$ Paul Vetschera, ${ }^{\dagger, \S}$ Christina Heichler, ${ }^{\%}$ Andriy Chmyrov $, \dagger, \S, \|$ Clemens Neufert, ${ }^{\%}$ Dierk Niessing, ${ }^{\times, \downarrow}$ Vasilis Ntziachristos, ${ }^{\dagger, \S, \|}$ and Andre C. Stiel* ${ }^{\dagger} \dagger$

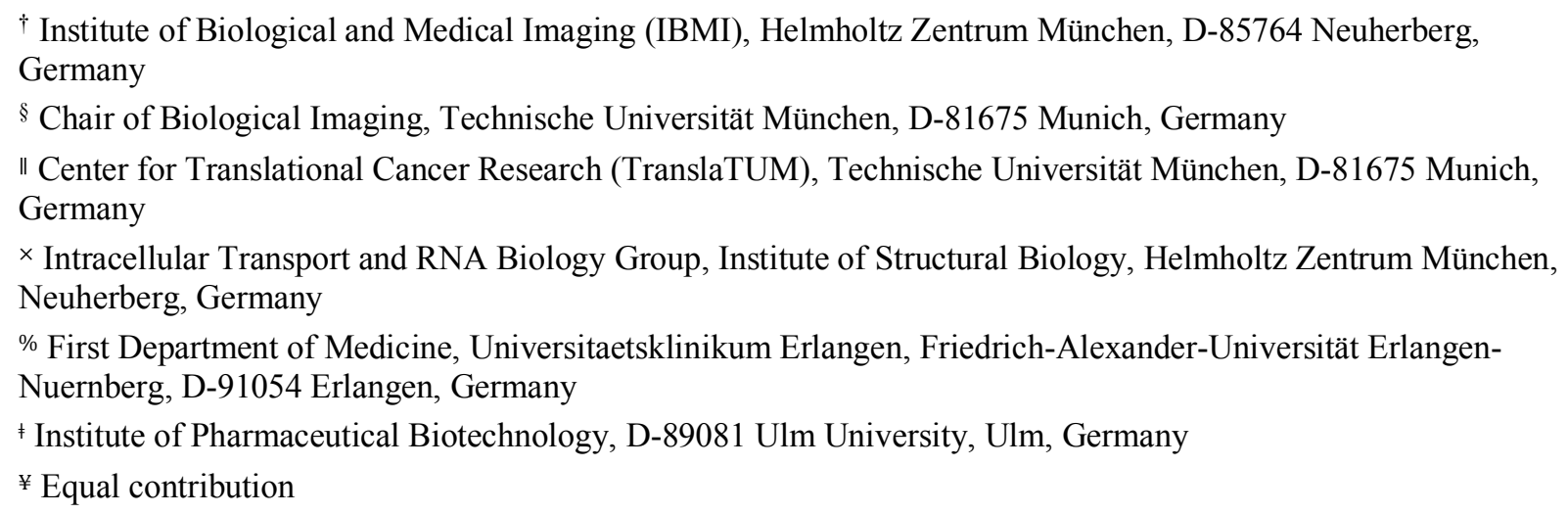

\section{Supplemental Information}

S-2 Supplementary Text 1: Extended Material and methods

S-7 Supplementary Table 1 Characteristics of dyes and proteins

S-8 Supplementary Figure 1 Photophysics of OA signal generation.

S-9 Supplementary Figure 2 Flow chart of laser pulse energy lookup table measurement

S-10 Supplementary Figure 3 Measure-and-fit scheme

S-11 Supplementary Figure 4 Flow chart showing the data acquisition

S-12 Supplementary Figure 5 Improvement in optoacoustic spectrum

S-13 Supplementary Figure 6 linear relationship of OAS and absorbance

S-14 Supplementary Figure 7 Jablonski energy diagram for monomers and geometrically perfect $\mathrm{H}$-type dimers of Methylene blue

S-15 Supplementary Figure 8 Bleaching measured as optoacoustic signal of dyes

S-16 Supplementary Figure 9 Optoacoustic signal for Rh800 at $695 \mathrm{~nm}$ and $635 \mathrm{~nm}$

S-17 Supplementary Figure 10 Segmented linear relation between absorbance and optoacoustic signal for ICG

S-18 Supplementary Figure 11 Linear relationship of OAS and absorbance for the main peaks S-7 and shoulders for proteins

S-19 Supplementary Figure $12 \mathrm{HcRed}$ Structure

S-20 Supplementary Table 2. Data collection and refinement statistics

S-21 Supplementary Figure 13 Single slices of ICG colon. 


\section{Supplementary Text 1: Extended Material and methods}

\section{Theory on optoacoustic generation efficiency (PGE)}

Vibrational relaxation (non-radiative energy decay) from an excited state produces a localized change in temperature, resulting in an optoacoustic signalvia thermal expansion. This change in pressure is generally described by equation [1] ${ }^{1}$.

$$
p_{0}=\Gamma \mu_{a} \varphi\left(1-\Phi_{f l}\right)
$$

In equation [1], $\mu_{a}$ is the local optical absorption coefficient, $\varphi$ is the local light fluence, and $\Phi_{\mathrm{fl}}$ is the fluorescence quantum yield. $\Gamma$ is the dimensionless Grüneisen parameter, which represents the thermoelastic properties of the medium. Equation [1] dismisses all other competitive channels, like conformational changes, photochemical transformations, intramolecular charge transfer, electron transfer, intersystem crossing, proton transfer, exciplex formation, excimer formation, and energy transfer $^{2}$ (Figure 1a). All of these processes reduce the number of available electrons that undergo immediate vibrational relaxation and produce a change in pressure ${ }^{3-5}$. Thus, in order to accurately compare different optoacoustic capacities, the OAS has to be normalized by the absorbance, so that the slope of the linear relation between the OAS and absorbance yields, the photoacoustic generation efficiency (PGE), which is a measure of how much of the absorbed energy is converted to pressure ${ }^{6-}$ 10. Photostability and the lack of any fluorescence make BBN an ideal reference for photoacoustic measurements; accordingly, we assign to it a PGE value of 1.0, assuming that no other decay channels take place ${ }^{9,10}$. After BBN normalization, equation [1] can be rewritten to derive equation [2]. Thus, PGE is attenuated by fluorescence $\left(\Phi_{f l}\right)$, and the energy 'stored' in long-lived transients such as a triplet state $\left(\Phi_{s t}\right)$ or any other photochemical decay pathway $\left(\Phi_{x}\right)^{11,12}$.

$$
\mathrm{PGE}=\frac{p_{0}}{\mu_{a}}=\Gamma \varphi\left(1-\Phi_{f l}-\Phi_{s t}-\Phi_{x}\right)
$$

\section{Multi-modal laser spectrometer (MLS) setup}

Building upon previous art ${ }^{13}$, we developed a multi-modal laser spectrometer (MLS, Suppl. Figure $1 \mathrm{~b}$ ) that allows us to record all three modalities (optoacoustics, absorbance, fluorescence) simultaneously in the wavelength range between 420-1000 $\mathrm{nm}$. Suppl. Figure $1 \mathrm{~b}$ schematized the optical system consisting of: 1. A diode-pumped solid-state optical parametric oscillator (DPSS OPO, SpitLight DPSS250 OPO, Innolas Laser GmbH, Germany) laser covering wavelength range $420-2000 \mathrm{~nm}$ with a $10-50 \mathrm{~Hz}$ pulse repetition rate (PRR), a pulse width at full width at half maximum $\tau_{F W H M}=7 \mathrm{~ns}$, and a $<1 \mathrm{~nm}$ linewidth of specific wavelength output. The OPO signal beam of 420-709 $\mathrm{nm}$ is output with horizontal polarization and idler beam 710-2000 nm with vertical polarization (switching controlled by a stepper motor and a microcontroller Arduino Uno via USB); 2. A pulse energy control to maintain constant pulse energy, which is measured using pyroelectric sensor head PE10-C and USB readout power meter Juno (Ophir-Spiricon LLC, USA), and controlled by using a combination of a motorized half-wave plate (HWP, AHWP05M-600; mounted on motorized rotation stage PRM1Z8; Thorlabs Inc., USA), a polarizing beam splitter (PBS052, Thorlabs Inc., USA), and a beam dump; 3 . An optical filter wheel that switches between long-pass (LP, cut-off wavelength at $700 \mathrm{~nm}$ to allow for OPO idler beam) and short-pass (SP, cut-off at $800 \mathrm{~nm}$ for signal beam) edge filters that are switched by an Arduino Uno via a stepper motor, in order to remove the possible leakage of signal/idler at output beam; 4 . An electrically controlled diaphragm shutter (SHB05T, Thorlabs Inc., USA) that is triggered by an Arduino Uno; And 5. a fiber bundle and its coupling lens (numerical aperture, NA 0.22; 430 fibers bundled in $\phi 4.6 \mathrm{~mm}$; CeramOptec $\mathrm{GmbH}$, Germany) to deliver light to a deionized water chamber which is determined for optimal coupling of optoacoustic wave.

In the water chamber, of which the walls were painted with black, we custom-built a holder to fix the relative position of all components, including: 1 . The IBIDI chips ( $\mu$-Slide I Luer, $200 \mu \mathrm{m}$ channel thickness, ibidi $\mathrm{GmbH}$, Germany) for sample and reference materials (water solution of Indian ink). The in-line reference chip is inserted in the beam path to remove the influence of laser pulse energy fluctuation of the sample spectrum. 2. Multi-mode fibers and collimating lens that deliver broadband light from a deuterium tungsten lamp (DH-2000-BAL, Ocean Optics, USA) and collect the transmitted light through the sample to a Vis-NIR spectrometer (USB4000-VIS-NIR, Ocean Optics, USA). Both the illumination and the detection fiber were attached to a collimator lens rotated by $45^{\circ}$ to the optoacoustic illumination-detection axis; And 3. an ultrasound transducer (V382-SU, central frequency $3.5 \mathrm{MHz}$, focus length $38.1 \mathrm{~mm}$, diameter $13 \mathrm{~mm}$ ) that simultaneously detects optoacoustic wave generated from 
reference and sample chips. The detected temporal optoacoustic signals were amplified by a $10-60 \mathrm{~dB}$ variable-gain amplifier (DHPVA, FEMTO Messtechnik GmbH, Germany) and sampled by a 12-bit digitizer (DAQ, Gage CSE1222, DynamicSignals LLC, USA). At each wavelength 3-10 sequences are acquired for signal averaging.

The data acquisitions using DAQ and Vis-NIR spectrometer were synchronized using either the trigger from the laser or the output of a photodetector (Si Biased Detector, DET36A/M, Thorlabs Inc., USA) induced by stray light in laser beam path. All data acquisition, processing and setup synchronization are controlled by our custom-built programs using Matlab 2015b (The MathWorks Inc., USA).

\section{Synchronization of signals measurement}

Empowered by the MLS spectrometer, four spectra of a sample can be obtained simultaneously: optoacoustics, absorbance, fluorescence excitation, and emission. 100-200 $\mu \mathrm{L}$ samples were filled in IBDI chips.

Since fluorescence and optoacoustic signal was excited by the same laser pulses, both can be recorded simultaneously. We set the Vis-NIR spectrometer in external triggering mode for excitation/emission spectrum measurement and shared the trigger as optoacoustic recording. When measuring optoacoustic spectrum and fluorescence spectra, the electrical shutter is opened, while the shutter to the lamp of Vis-NIR spectrometer is closed, and the ultrasound transducer, DAQ, and spectrometer (configured to external triggering mode and fixed integration time of $10-90 \mathrm{~ms}$ ) are triggered by the laser or a photodiode to acquire data. Due to the coincidence of sweeping excitation laser and fluorescence emission, there was overlapping region of the two in a direct recording. To extract the excitation spectrum only, for example, from the recordings, we used the normalized readings of three different emission wavelengths at each excitation wavelengths. When the excitation wavelength was overlapping with one of the selected emission wavelengths, we ignored the current emission reading and used the extrapolated value averaged from the other two emission wavelengths. Similar method was adopted to extract the emission spectrum from such overlapping recordings.

When measuring absorbance, only the Vis-NIR spectrometer acquires data (configured to circularbuffered acquisition mode using integration time that uses $90 \%$ of the full pixel well depth when making dark/no sample measurement), and laser light is blocked by the closed shutter and lamp shutter is opened to deliver broadband white light to the sample. The setup has a time delay for the absorption measurement of at least $110 \mathrm{~ms}$ after the laser pulses due to shutter responses.

\section{Lookup table for constant pulse energy}

One challenge in measuring with high-fidelity on common dyes/proteins based chromophores is that they suffer from photobleaching and such processes are usually wavelength dependent. Constant pulse energy was ensured by the use of half-wave plate on motorized rotation stage and a polarizing beam splitter; using a lookup table and adapting the polarization with the half-wave plate, we kept the power constant at $1.2 \mathrm{~mJ}$ for the proteins measurements and $1 \mathrm{~mJ}$ for the dyes, with root-mean-squared error (RMSE) $2 \%$ over the whole illumination spectrum. We confirmed the linearity and stability of the optoacoustic signal generated by $\mathrm{BBN}$ and $\mathrm{NiCl}_{2}$ in the range of fluences used in our experiments.

Thus to ensure a controlled constant delivery of pulse energy to the sample across the $420-1000 \mathrm{~nm}$ range, the operation of MLS starts with a pulse energy lookup table measurement. Suppl. Figure 2 shows a complete flow chart on our high-precision laser pulse energy lookup table measurement method. The lookup table is intended to reflect the sinusoidal oscillation relation between the angle difference of HWP/PBS and the pulse energy of OPO at certain wavelengths. Therefore, we used a measure-and-fit scheme (Eq. 3 ) to fasten the speed of lookup table measurement.

$$
E(\lambda, \theta)=A_{0}(\lambda) \cdot\left[\frac{1}{2}+\sin \left(\frac{2 \pi}{180^{0}} \theta+\theta_{0}\right)\right],
$$

where $E(\lambda, \theta)$ is the pulse energy measured at laser wavelength when HWP is at angle $\theta ; A_{0}(\lambda)$ is the maximum pulse energy that is achievable only when HWP angle is at $\theta_{0}$ that coincides with the transmission polarization of PBS.

According to Eq. 3 (shown in Suppl. Figure 2), we first measured the energy at one OPO signal wavelength, e.g., at $420 \mathrm{~nm}$ with HWP angle rotating with step $2^{\circ}$ across $72^{\circ}$ angles (in order to include one peak and one trough). Then we fitted the energy-angle curve to the parametric sinusoidal model as indicated by Eq. 3, to find the phase angle $\theta_{0}$ where the peak energy appears. Fixing HWP at the peak phase angle $\theta_{0}$, we continued to measure the peak energy $A_{0}(\lambda)$ of the rest signal wavelengths, and based on which we extrapolated the sinusoids model. We showed in Suppl. Figure 3a an exemplary laser pulse energy lookup table measured using our measure-and-fit based scheme. The same procedure was applied to OPO idler wavelengths $(\geq 710 \mathrm{~nm})$ to obtain a crude estimate of the lookup 
table. But due to the fluctuation of OPO laser pulse, especially at wavelengths around $710 \mathrm{~nm}$ where signal/idler beam are separated, the extrapolated lookup table required still correction. Aiming at a pulse energy of e.g. $1.0 \mathrm{~mJ}$ at all wavelengths, we measured the pulse energy back using the angles suggested by the lookup table targeting at $1.0 \mathrm{~mJ}$, and obtained the ratio of the targeted energy and the new measurements. Then we multiply this ratio by the amplitude $A_{0}(\lambda)$ to scale the lookup table proportionally. Usually, less than 5 iterations of this lookup table correction would enable a RMSE of $<2 \%$ at a specified pulse energy, as shown in Suppl. Figure 3b.

\section{Principle of in-line correction}

Another challenge resides with the pulse-to-pulse fluctuation at given wavelength after homogenizing the laser pulse energy, which can range from a few percent to more than $30 \%$ as at the transition wavelengths of signal/idler output (Suppl. Figure $2 \mathrm{~b}$ ). However, the response of the power meter is too low to ensure the correction of pulse-to-pulse energy fluctuations. Furthermore, while the peak power variations might be correctible with a fast-response power meter, the variation in pulse width is harder to measure and correct with high-precision.

To minimize the influence of such fluctuation, we designed an in-line reference using water solution of Indian ink in optoacoustic sensing. The resulting OA signals from both reference and sample are collected by the ultrasound transducer in the same temporal sequence. As shown in Figure $1 \mathrm{~b}$, laser light fluence $\varphi_{0}$ with fluctuation $\Delta f l$ traveling a water path $d_{F R}$ from fiber to reference chip pass through the low concentration of ink with a light fluence $\varphi_{R}$ according to Beer-Lambert's law:

$$
\varphi_{R}=e^{-d_{F R} \cdot \mu_{H 2 O} O} \cdot \varphi_{0}(1+\Delta f l)=e^{-d_{F R} \cdot \mu_{H 2 O} O} \cdot \varphi_{0} \cdot \frac{(1+\Delta E) \cdot E_{0}}{\left[(1+\Delta \sigma) \cdot \sigma_{0}\right]^{2}},
$$

where $\mu_{H 2 O}$ is water absorbance. It is important to note that the pulse-to-pulse light fluence fluctuation $\Delta f l$ manifests as both a peak power $\left(E_{0}\right)$ variation $(\Delta E)$ and a pulse width $\left(\sigma_{0}\right)$ variation $(\Delta \sigma)$, assuming Gaussian temporal profile. The pulse width variation (sub-nanosecond for $7 \mathrm{~ns}$ pulse width we used) is a term cannot be corrected using a slow-responding power meter or photodiode for optoacosutics, but only possible by using high-speed photodiode ( $<100$ picosecond) and fast acquisition system $(>20$ $\mathrm{GHz}$ ), which is a costly. Instead, we used optoacoustic reference. The transmitted light after reference chip with absorbance $\mu_{a R}$ and path $d$ same to channel thickness of IBIDI chip then travels a water path $d_{R S}$ from reference chip to sample chip. The incidence light fluence to the sample, $\varphi_{S}$, is:

$$
\varphi_{S}=e^{-d \cdot \mu_{a R}} \cdot e^{-d_{R S} \cdot \mu_{H 2 O}} \cdot \varphi_{R}
$$

The photoacoustic signal generation induced by optical absorption and the subsequent thermal expansion can be described by a simplified model ${ }^{14}, p_{0}=\Gamma \mu_{a} \varphi$, assuming a linear relation between absorption coefficient and light fluence in low absorbance and low scattering sample ${ }^{15}, \Gamma$ is the Grüneisen coefficient indicating the conversion efficiency from heat to pressure wave. Therefore, the optoacoustic wave generated by the reference chip is:

$$
p_{R}=\Gamma_{R} \cdot \mu_{a R} \cdot \varphi_{R}
$$

Similarly, the sample generated optoacoustic wave is:

$$
p_{S}=\Gamma_{S} \cdot \mu_{a s} \cdot \varphi_{S}
$$

Dividing sample optoacoustic signal by reference can cancel the influence of laser pulse-to-pulse fluctuation if substituting in the light fluence to reference (Eq. 4) and sample (Eq. 5).

$$
\frac{p_{S}}{p_{R}}=\frac{\Gamma_{S} \cdot \mu_{a S} \cdot \varphi_{S}}{\Gamma_{R} \cdot \mu_{a R} \cdot \varphi_{R}}=\Gamma_{S / R} \cdot \frac{\mu_{a S}}{\mu_{a R}} \cdot e^{-d \cdot \mu_{a R}} \cdot e^{-d_{R S} \cdot \mu_{H 2 O}}
$$

As a consequence, Eq. 8 indicates that fluctuation correction renders a mixed spectrum of the reference and sample. The first term on the right-hand side of Eq. 8 is a ratio of Grüneisen coefficients of sample over reference. The second term is absorption coefficient ratio of sample over reference. The third term indicates the spectral coloring effect ${ }^{16}$ (light fluence alteration) by the in-line reference. The fourth term represents the spectral coloring by the water path between reference and sample chips.

There are ways to account for the effect of above-mentioned terms. The absorption coefficient $\mu_{a R}$ of reference solution can be obtained by measuring its absorbance $\mathrm{A}\left(\mathrm{cm}^{-1}\right)$ and converting using $\mu_{a R}=\ln (10) \cdot A$, assuming low scattering by a low concentration (i.e. absorbance) of ink. Thus multiplying the spectrometer measured $\mu_{a R}$ and Eq.8 would remove the influence by ink spectra. Furthermore, although the effect is minor, the spectral coloring effect by ink can be removed, given the thickness of IBIDI chip channel thickness. Similarly, given water path $d_{R S}$ between reference and sample chip and water absorption coefficient (data adapted from http://www.spectra.arizona.edu/), the spectral coloring effect by water can be removed. The corrected optoacoustic signal at wavelength as a result is:

$$
p_{S}(\lambda)=\Gamma_{S / R} \cdot \mu_{a S}(\lambda)
$$

where the Grüneisen coefficient ratio $\Gamma_{S / R}$ is not supposed to be wavelength dependent thus a scaling factor of optoacoustic signal amplitude. For reproducible comparison across various samples, 
if we use ink as reference for all the samples, the Grüneisen coefficient ratio still reflects the heat conversion efficiency of samples.

\section{Data process for multi-modal measurement}

Suppl. Figure 4 shows the program for data acquisition and data process in multi-modal laser spectroscopy. According to our in-line referencing to correct for the laser pulse-to-pulse fluctuation, we applied similar process to fluorescence emission data as to optoacoustic data.

The computation of the spectra (blue shading part showed in Suppl. Figure 4) is done as described in the following sequential steps: taking a sample of e.g. $\mathrm{NiCl}_{2}$ with $0.5 \mathrm{OD}$ at $720 \mathrm{~nm}$. The results are shown in Suppl. Figure 5, providing an examination on the improvement brought by each of the processes in comparison with the raw spectrum - Raw (Max) in Suppl. Figure 5 - which is acquired by taking simply the maximum value measured at each wavelength upon homogenized laser pulse energy. Matching the absorbance spectrum, the Raw spectra showed a coefficient of determination $R^{2}$ of 0.988 and a standard deviation $7.9 \%$ between repeated recording, owing to the $2 \%$-RMSE laser energy control (Suppl. Figure 3).

1. 'Filtered'. We firstly filtered the acquired temporal sequence by using a fourth order digital bandpass finite impulse response filter in Fourier frequency band $0.1-10 \mathrm{MHz}$ respecting the bandwidth of our transducer, a 1-D digital wavelet transform (decomposition level=5) based denoising method to reduce thermal noise level, a 3rd order Savitzky-Golay filter with smoothing length corresponding to $20 \mathrm{MHz}$ in our DAQ (200 MSamples/second) to reduce digitization error.

2. 'Hilbert'. Hilbert transform is used to detect the peak envelope of temporal sequence. The optoacoustic spectrum relies on the maximum signal in the sequence at the position (time-offlight) of sample and reference. We detected the peak signals by summing up all sequences measured at all wavelengths, then finding the locations of the peaks of sample and reference respectively, and using the peak locations as center point to take averaged values of adjacent 3-5 sample points from sequence of each wavelength. A better signal-to-noise ratio (SNR) of optoacoustic spectra for low absorbance sample was achieved using this way than taking area under the curve of signal envelope.

3. '- Dark'. We subtracted from the raw signal with the root mean square of a dark background signal that was measured with no laser irradiation before each spectral measurement to reduce the noise floor and thus improve the detection sensitivity of low concentration sample.

4. 'Pulse Fl.' To reduce the influence of laser pulse-to-pulse energy fluctuation, we divided the sample spectrum acquired at each laser pulse by the corresponding reference spectrum. But this step introduces spectra of ink into the sample spectra.

5. '- Ink Spect.' To remove the ink spectra, we multiplied the fluctuation corrected spectra with normalized spectra of ink to recover the sample spectra.

6. '- Coloring'. Notwithstanding that it is minor effect in 420-900 $\mathrm{nm}$ range induced by the spectral coloring by ink (short absorption path) and water path between reference and sample (water absorbance at $900 \mathrm{~nm}$ is $0.025 \mathrm{~cm}^{-1}$ ), we corrected for spectral colorings. The improvement was neither reflected in the coefficient of determination $\mathrm{R}^{2}$ nor standard deviation of measurements, but was instead shown as a higher fidelity in the peak value at $420 \mathrm{~nm}$ as shown in supplementary Figure 5.

\section{MLS spectra quality validation}

To validate the optics setup and the correction steps, we analyzed the spectra of $\mathrm{NiCl}_{2}$. $\mathrm{NiCl}_{2}$ is known to have no apparent photo-fatigue and a complete transfer of absorbed energy into acoustic waves via non-radiative decay channels, so its absorption spectrum should correlate with its optoacoustic spectrum ${ }^{17}$. The OA spectra of $\mathrm{NiCl}_{2}$ measured without any correction has a correlation coefficient of $\mathrm{R}^{2}=0.988$ and a standard deviation $\mathrm{SD}=7.9 \%$ while using the full correction protocol of our MLS, the correlation coefficient and standard deviation are respectively $R^{2}=0.997$ and $S D=1.9 \%$. The improvement of the spectral quality through our measurement protocol is significant over a range of concentrations, with an $\mathrm{R}^{2}$ of 0.85 for a $\mathrm{NiCl}_{2}$ sample with $\mathrm{OD}$ as low as 0.05 (Suppl. Figure 1c-e). In order to test the wavelength accuracy of our setup, we measured OA and absorbance spectra of $\mathrm{KMnO}_{4}$ (Suppl. Figure 1f), and we were able to match its six absorption peaks with $2 \mathrm{~nm}$ accuracy correctly. To further characterize our MLS system, we similarly measured different concentrations of Brilliant Black N dissolved in PBS (BBN, Suppl. Figure $1 \mathrm{~g}$ and $\mathrm{h}$ ). $\mathrm{BBN}$, similar to $\mathrm{NiCl}_{2}$, has a negligible fluorescence quantum yield $\left(Q Y_{\text {fluo }}\right)$ and very high photostability, and thus undergoes fully non-radiative deexcitation. Importantly, $\mathrm{BBN}$, in contrast to $\mathrm{NiCl}_{2}$, is soluble and stable in aqueous buffer solutions. BBN thus shares a common Grüneisen parameter ${ }^{9,10,17}$ with labels for in vivo imaging, which are frequently dissolved in 
aqueous buffer systems. The combination of the excellent solubility of BBN and its stable generation of OA signals over a range of concentrations and excitation energies makes it well suited to serve as a universal standard for precise and reproducible OA measurements. Such characteristics allow reproducible characterization of dyes and other labels eventually allowing the determination and reporting of universally meaningful OA signal strength values.

\section{Visible MSOT Images:}

Dextran sodium sulphate (DSS) colitis model and sample preparation:

Colon inflammation was induced in reporter mice expressing tdTomato under control of the collagen VI promoter (tdTomatoColVI) by $2.5 \%$ (w/vol) DSS (MP Biomedicals) in drinking water for 7 days as previously described (Wirtz et al. 2007). Mice were euthanized at day 8, when the entire colon was removed and rinsed with PBS (Sigma-Aldrich). The colon was incubated in Roti-Histofix $4.5 \%$ (Carl Roth) overnight at $4^{\circ} \mathrm{C}$, before samples were proceeded to the measurements.

Uninflamed or DSS-induced, inflamed colons and tube containing ink were placed in agar bed. The sample was illuminated by nanosecond excitation pulses at $1 \mathrm{~Hz}$ generated by an optical parametric oscillator (OPO) laser (Spitlight-DPSS 250 ZHG-OPO, InnoLas) delivered by a fiber illuminating the sample at an angle of $45^{\circ}$. Constant pulse energy was maintained as describe before. Data was averaged over 3 laser pulses to improve the signal-to-noise ratio. We record spectral images from 420 to $695 \mathrm{~nm}$ every $5 \mathrm{~nm}$ before and after 80 cycles of 100 pulses of 480 and $550 \mathrm{~nm}$ light. Detection: Ultrasonic detection was performed using a curved array made of 64 elements encompassing a circle with a mechanical focal distance of $4 \mathrm{~cm}$ and an angular coverage of $172^{\circ}$. The array had a central frequency of $5 \mathrm{MHz}$ (Imasonic SAS, Voray, France). Both the transducer array and fiber outputs were submerged in a water bath; the laser beams and ultrasonic transducer array are in fixed position for all data acquisitions. Ultrasound data was reconstructed to images using a model-based approach.

\section{ICG MSOT Images:}

MSOT data acquisition, reconstruction, and unmixing

All MSOT data has been recorded using the MSOT inVision 256 (iThera Medical GmbH, Munich, Germany). MSOT spectra between 680 and $900 \mathrm{~nm}$ of freshly euthanized mouse filled rectally with ICG solution $65 \mu \mathrm{M}$ were recorded. IThera commercial software ViewMSOT was used for image reconstruction and spectral unmixing. AMF spectral unmixing was performed using ICG absorption spectrum and each of our ICG optoacoustic spectrums.

\section{Elucidation of HcRed structure:}

Crystallization, diffraction data collection and processing

HcRed was purify as describe above. The pure protein was exchange from PBS to TRIS buffer pH8.0 containing $300 \mathrm{mM} \mathrm{NaCl}$ and concentrate until $5 \mathrm{mg} / \mathrm{ml}$.

The crystallization experiments for HcRed were performed at the X-ray Crystallography Platform at Helmholtz Zentrum München. The initial crystallization screening was done at $292 \mathrm{~K}$ using $5 \mathrm{mg} / \mathrm{ml}$ of protein with a Mosquito (SPT Labtech) nanodrop dispenser in sitting-drop 96-well plates and commercial screens. After selecting the best hits from the screening, manual optimization was performed. The monomeric HcRed crystals grew in $0.1 \mathrm{M} \mathrm{BICINE} \mathrm{pH} \mathrm{8.5,22 \%} \mathrm{w/v} \mathrm{Polyethylene} \mathrm{glycol} \mathrm{10,000.} \mathrm{For} \mathrm{the}$ $\mathrm{X}$-ray diffraction experiments, the crystals were mounted in a nylon fiber loop and flash-cooled to $100 \mathrm{~K}$ in liquid nitrogen. The cryoprotection was performed for $\sim 2$ seconds in reservoir solution complemented with $25-30 \%(\mathrm{v} / \mathrm{v})$ ethylene glycol. Diffraction data were collected on the SLS PXIII X06DA beamline (PSI, Villigen) and the measurements were performed at $100 \mathrm{~K}$. X-ray diffraction data set for HcRed was collected to $2.3 \AA$ resolution (Suppl. Table 2). The data set was indexed and integrated using XDS ${ }^{18}$ and scaled using SCALA ${ }^{19,20}$. Intensities were converted to structure-factor amplitudes using the program TRUNCATE ${ }^{21}$. Suppl. Table 2 summarizes data collection and processing statistics.

\section{Structure determination and refinement}

The structure of HcRed was solved by the MolRep 20 program from $C C P 4^{22}$. As a search model the closest HcRed homologue served (PDB ID: 1yzw) ${ }^{23}$. Model rebuilding was performed in $\mathrm{COOT}^{20}$. The further refinement was done in REFMAC5 using the maximum-likelihood target function. The stereochemical analysis of the final model was done in $\mathrm{PROCHECK}^{24}$ and MolProbity ${ }^{25}$. The final model is characterized by $\mathrm{R} / \mathrm{R}_{\mathrm{free}}$ factors of $16.8 \% / 24.7 \%$ (Suppl. Table 2). Atomic coordinates and structure factors have been deposited in the Protein Data Bank under accession code 6Y1G. 
Supplementary Table 1 Characteristics of dyes (a) and proteins (b)

Supplementary Table 1a

\begin{tabular}{|c|c|c|c|c|c|c|c|c|c|c|}
\hline & $\begin{array}{l}\text { Abs } \\
\text { Max1, } \\
\mathrm{nm}\end{array}$ & $\begin{array}{l}\text { Abs } \\
\text { Max2, } \\
\mathrm{nm}\end{array}$ & $\begin{array}{c}\text { Abs } \\
\text { Max1, } \\
\mathrm{nm}\end{array}$ & $\begin{array}{c}\text { OAS } \\
\text { Max2, } \\
\mathrm{nm}\end{array}$ & $\begin{array}{l}\text { Ex } \\
\text { Max, } \\
\mathrm{nm}\end{array}$ & $\begin{array}{l}\text { Em } \\
\text { Max, } \\
\mathrm{nm}\end{array}$ & $\begin{array}{l}\text { Linear Fit at } \\
\text { Max1 (PGE) }\end{array}$ & $\begin{array}{l}\text { Linear Fit at } \\
\text { Max1 (PGE) }\end{array}$ & $\begin{array}{l}\text { Bleaching at } \\
\text { Max } 1^{b}\end{array}$ & $\begin{array}{l}\text { Bleaching at } \\
{\text { Max } 2^{\mathrm{b}}}\end{array}$ \\
\hline$M B$ & 615 & 665 & 610 & 665 & 665 & 680 & $\mathbf{0 . 6 1 X}-0.05$ & $0.37 x-0.07$ & $0.73 \pm 0.01$ & $0.85 \pm 0.01$ \\
\hline Rh800 & 630 & 690 & 635 & 695 & 690 & 705 & $1.14 X-0.43$ & $1.08 x-0.40$ & $0.95 \pm 0.02$ & $0.96 \pm 0.02$ \\
\hline Al750 & 698 & 750 & 696 & 740 & 745 & 770 & $0.85 x-0.04$ & $0.72 x-0.14$ & $0.85 \pm 0.02$ & $0.53 \pm 0.02$ \\
\hline $800 \mathrm{CW}$ & 710 & 775 & 710 & 770 & 770 & 789 & $0.86 X-0,04$ & $0.63 x-0.02$ & $0.63 \pm 0.02$ & $0.5 \pm 0.01$ \\
\hline
\end{tabular}

\section{Supplementary Table 1b}

\begin{tabular}{|c|c|c|c|c|c|c|c|c|c|}
\hline & $\begin{array}{l}\text { Abs Max1, } \\
\mathrm{nm}\end{array}$ & $\begin{array}{c}\text { Abs Max2, } \\
\mathrm{nm}\end{array}$ & $\begin{array}{c}\text { OAS Max1, } \\
\mathrm{nm}\end{array}$ & $\begin{array}{c}\text { OAS Max2, } \\
\mathrm{nm}\end{array}$ & Ex Max, nm & Em Max, nm & $\begin{array}{c}\text { Fluo } \\
\text { Quantum } \\
\text { Yield }\end{array}$ & $\begin{array}{l}\text { Linear Fit } \\
\text { at Max1 } \\
\left(_{(\mathrm{PGE})^{\mathrm{a}}}\right.\end{array}$ & $\begin{array}{l}\text { Linear } \\
\text { Fit at } \\
\text { Max2 } \\
(\text { PGE) }\end{array}$ \\
\hline sfGFP & - & 490 & 425 & 490 & $* * *$ & 503 & 0.65 & $\begin{array}{c}0.78 X- \\
0.01\end{array}$ & $\begin{array}{l}0.34 X- \\
0.05\end{array}$ \\
\hline mKate2 & 555 & 585 & 550 & 580 & 590 & 615 & 0.4 & $0.64 X+0.01$ & $\begin{array}{c}\mathbf{0 . 5 1 X + -} \\
0.03\end{array}$ \\
\hline mCherry & 545 & 585 & 545 & 580 & 585 & 610 & 0.22 & $\begin{array}{c}\mathbf{0 . 5 4} X+ \\
0.01\end{array}$ & $\begin{array}{c}0.36 \mathrm{X}- \\
0.01\end{array}$ \\
\hline tdTomato & 485 & 555 & 485 & 565 & 555 & 580 & 0.69 & $\begin{array}{c}\mathbf{0 . 8 0 X}- \\
0.02\end{array}$ & $\begin{array}{c}\mathbf{0 . 8 3} * \mathrm{X}- \\
0.08\end{array}$ \\
\hline HcRed & 570 & 590 & 570 & 590 & 590 & 635 & 0.015 & $\begin{array}{c}0.97 X- \\
0.01\end{array}$ & $\begin{array}{c}0.80 \mathrm{X}- \\
0.03\end{array}$ \\
\hline SMURFP & 600 & 645 & 600 & 645 & 645 & 666 & 0.18 & $\begin{array}{c}\mathbf{0 . 5 8 X}- \\
0.01\end{array}$ & $\begin{array}{c}0.55 X- \\
0.01\end{array}$ \\
\hline IRFP720 & 650 & 705 & 650 & 700 & 705 & 720 & 0.06 & $0.97 X-0.09$ & $\begin{array}{c}0.86 \mathrm{X}- \\
0.01\end{array}$ \\
\hline
\end{tabular}

a. Linear Fit of absorbance versus corrected optoacoustic signal, PGE: Optoacoustic generation efficiency. b. Bleaching rate after 5000 exaphotons. 
$a_{s}$
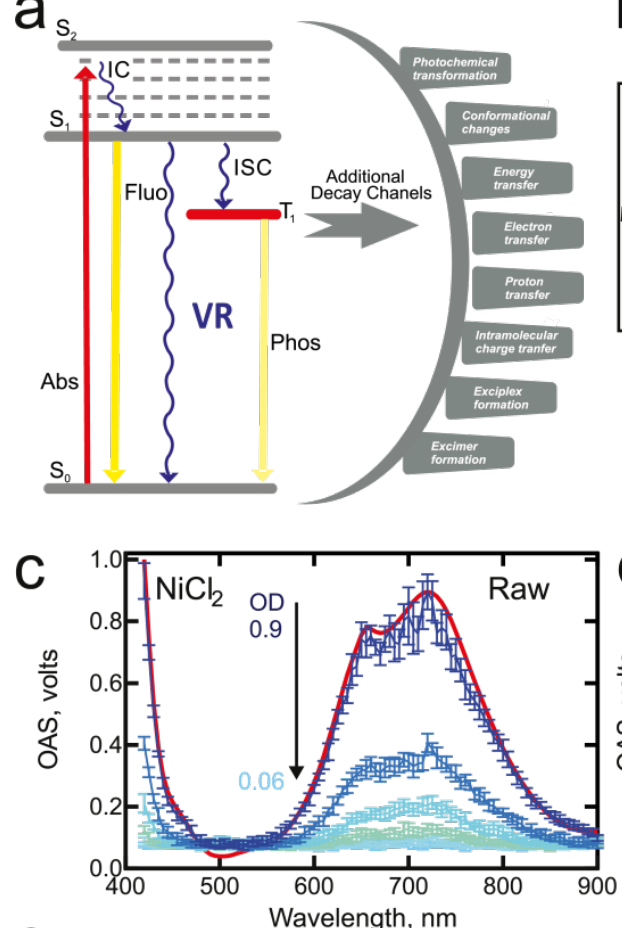

\section{e}

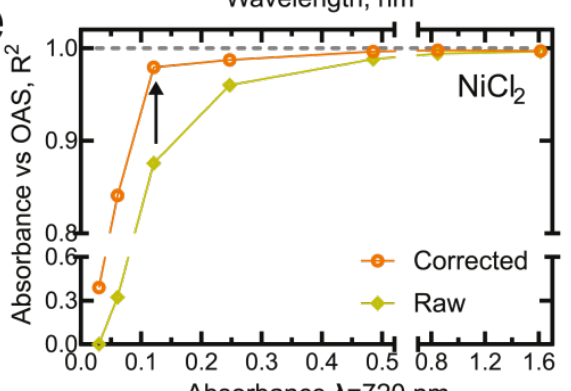

g

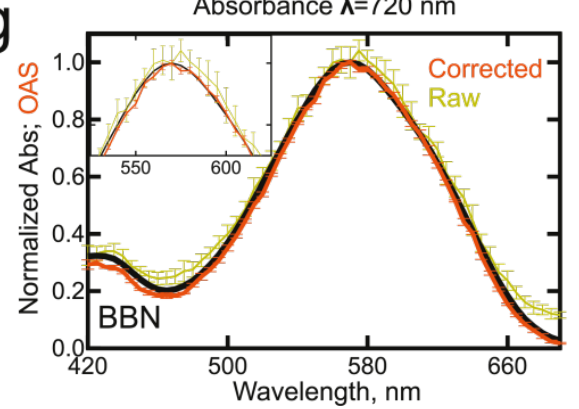

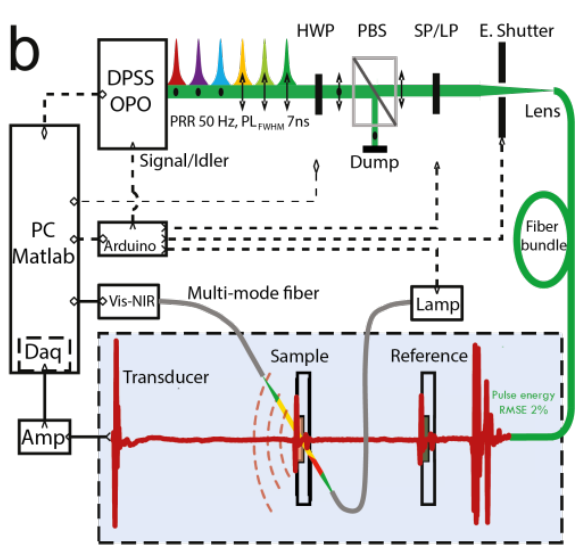
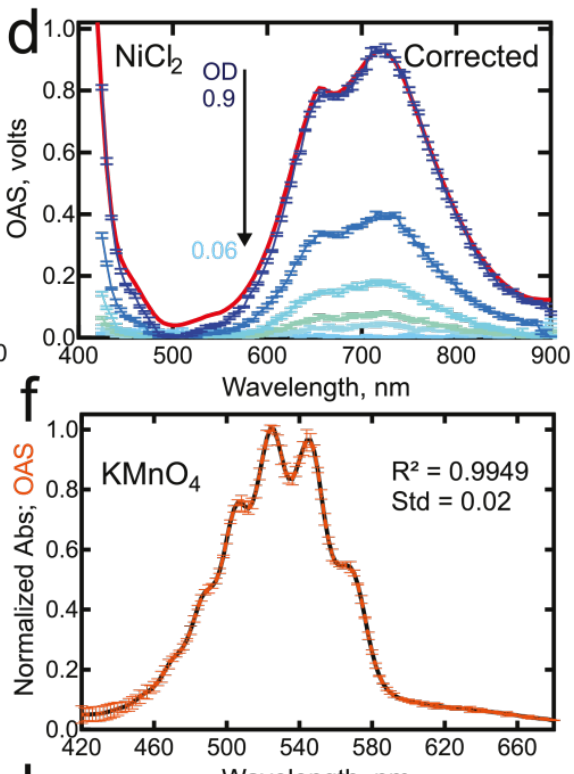

$\mathrm{h}$

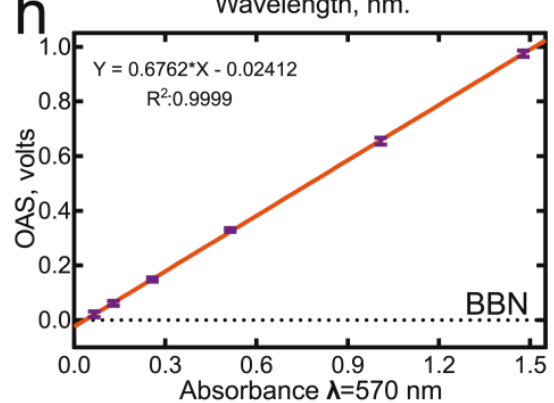

Supplementary Figure 1 (a) Photophysics of OA signal generation. Jablonski energy diagram. Plain lines represent radiative transitions, dashed lines represent nonradiative processes. Abs: absorbance, $\mathrm{S}_{0}$ : ground state, $\mathrm{S}_{1}$ : singlet excited state, $\mathrm{T}_{1}$ : primary triplet excited state, VR: vibrational relaxation, ISC: intersystem crossing, IC: internal conversion, Pho: phosphorescence, Fluo: fluorescence. (b) Setup diagram (explanations and abbreviations can be found in the text). (c) Raw optoacoustic spectrum of $\mathrm{NiCl}_{2}$ at different concentrations and (d) after correction. (e) The coefficients of determination of linearity between absorbance and optoacoustic spectrum of $\mathrm{NiCl}_{2}$ at decreasing concentrations. (f) $\mathrm{OA}$ and absorbance spectrum for potassium permanganate at $2 \mathrm{~nm}$ steps. (g) Raw and corrected optoacoustic spectrum of Brilliant black BN (BBN). (h) Linear relation between absorbance and OA signal for BBN. 
Measure and fit lookup table

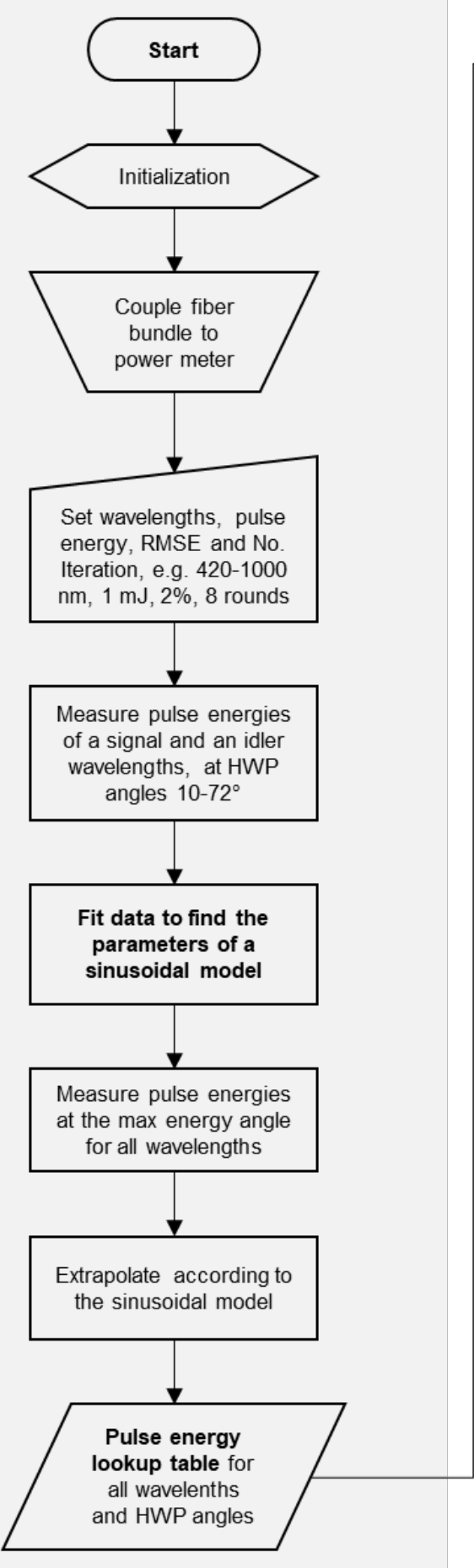

Iteration based precision improve

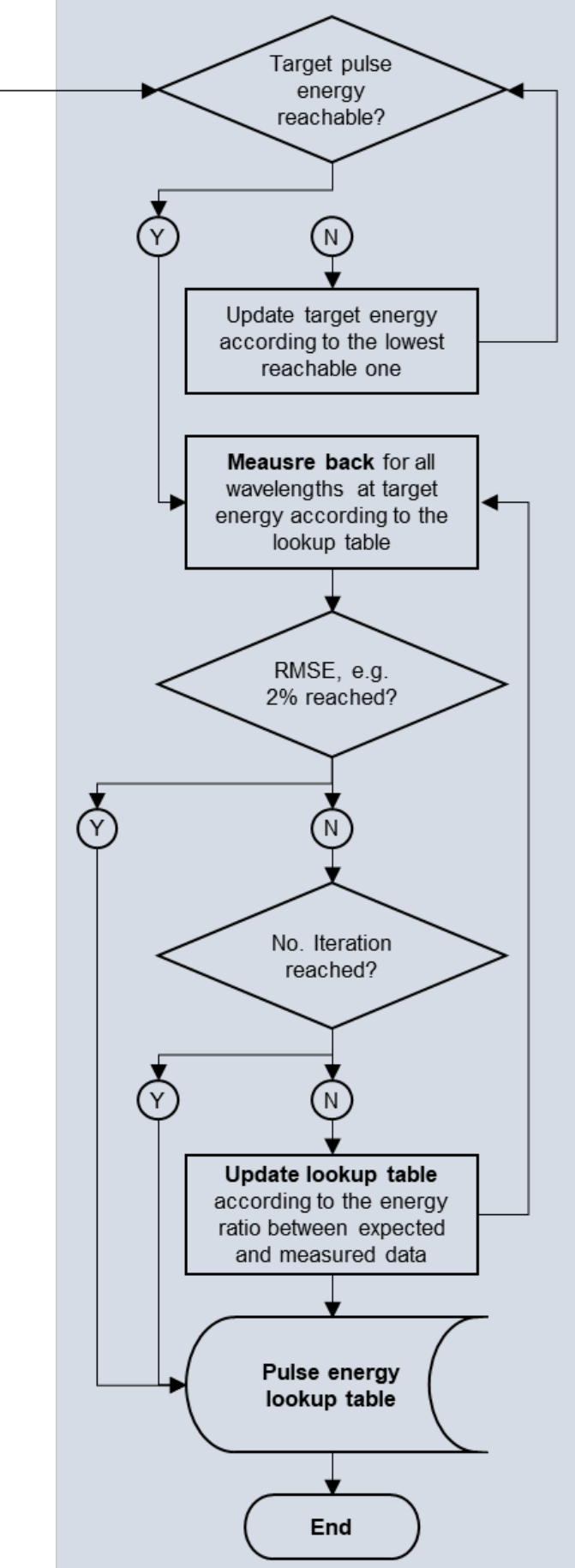

Supplementary Figure 2 Flow chart of laser pulse energy lookup table measurement. In grey shading (left) is the measure-and-fit scheme measuring laser pulse energy controlled by rotary half-wave plate and polarizing beam splitter. In blue shading (right) is the iteration-based pulse energy precision improvement method. 

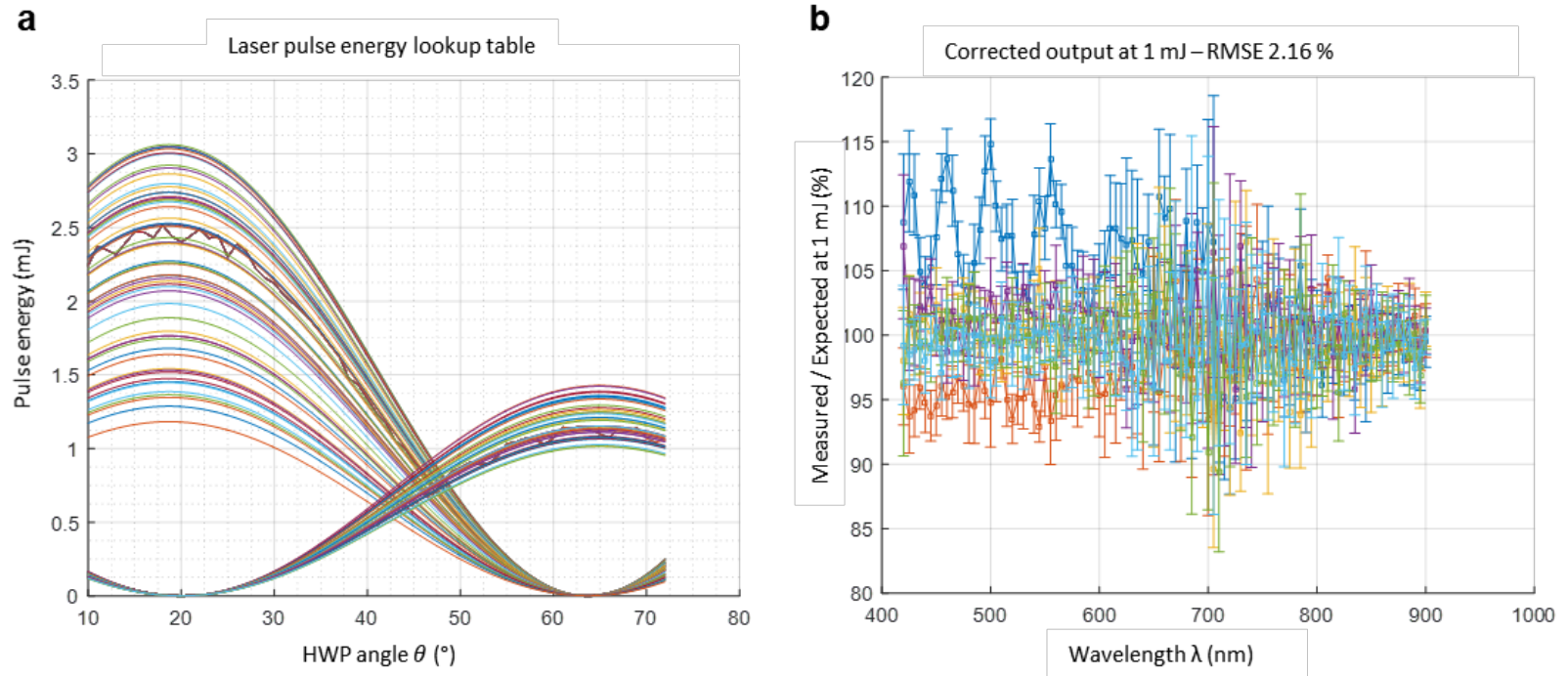

Supplementary Figure 3 (a) Measure-and-fit scheme measuring laser pulse energy controlled by rotary half-wave plate and polarizing beam splitter. (b) Iterated pulse energy lookup table correction. 


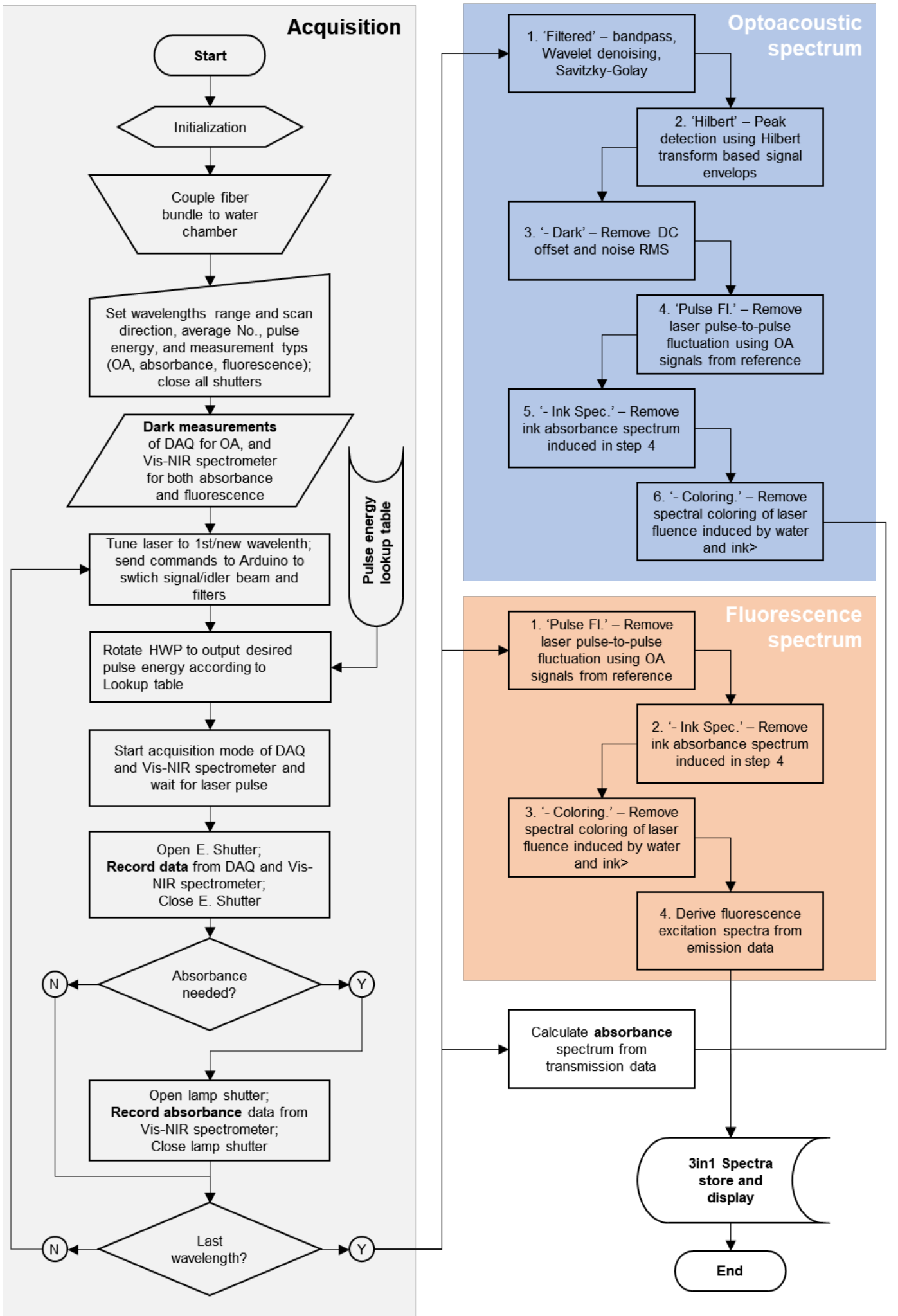

Supplementary Figure 4 Flow chart showing the data acquisition (in grey shading, left) and data process scheme (right; in blue for optoacoustic spectrum; in orange for fluorescence excitation and emission spectrum; absorbance is showed without special marks). Data input showed as 'Pulser energy lookup table' is imported as a result of measurements showed in Supplementary Figure 1. 


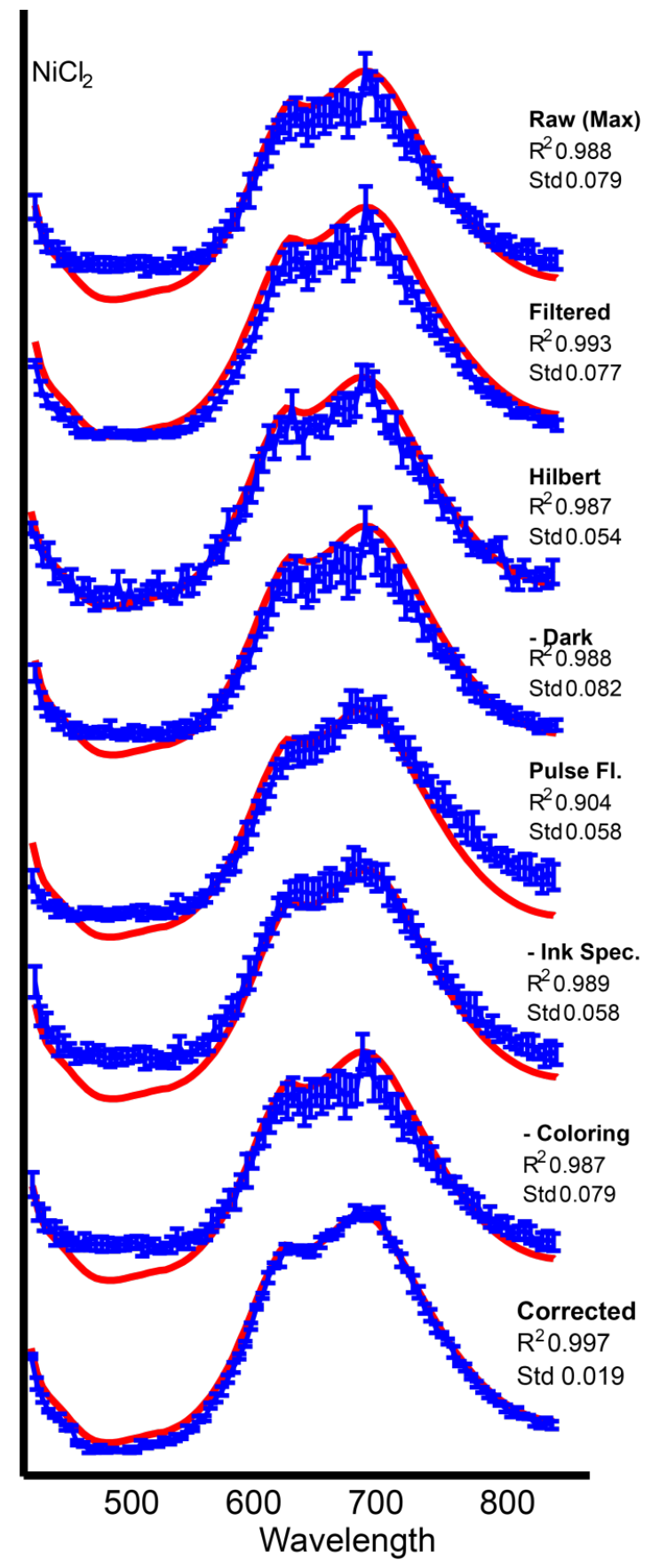

Supplementary Figure 5 Improvement in optoacoustic spectrum of $\mathrm{NiCl}_{2}$ brought by each of the correction steps. The coefficient of determination $R^{2}$ and standard deviation (Std) between measurements are shown to indicate the improvement by each of steps. 'Max (Raw)' shows a spectrum that can be obtained using simply the maximum values in sample optoacoustic signals generated by homogenized laser energy across wavelength. 'Filtered' shows the spectrum can be obtained using filtering techniques on signals. 'Hilbert' shows the spectrum formed by maximum values of the signal envelops using Hilbert transform. '- Dark' shows the spectrum that is without DC bias by subtracting values measured in dark (no laser). 'Pulse Fl.' shows the spectrum after pulse-to-pulse fluctuation correction enabled by our in-line reference. '- Ink Spec.' shows the spectrum after removing the ink spectrum which is induced when performing fluctuation correction. '- Coloring' shows the spectrum after the correction for the light fluence coloring effect induced by the reference Ink and US-couplant water. 'Corrected' shows the spectrum that can be obtained after performing all the afore-mentioned steps (further explanations can be found in the text). 

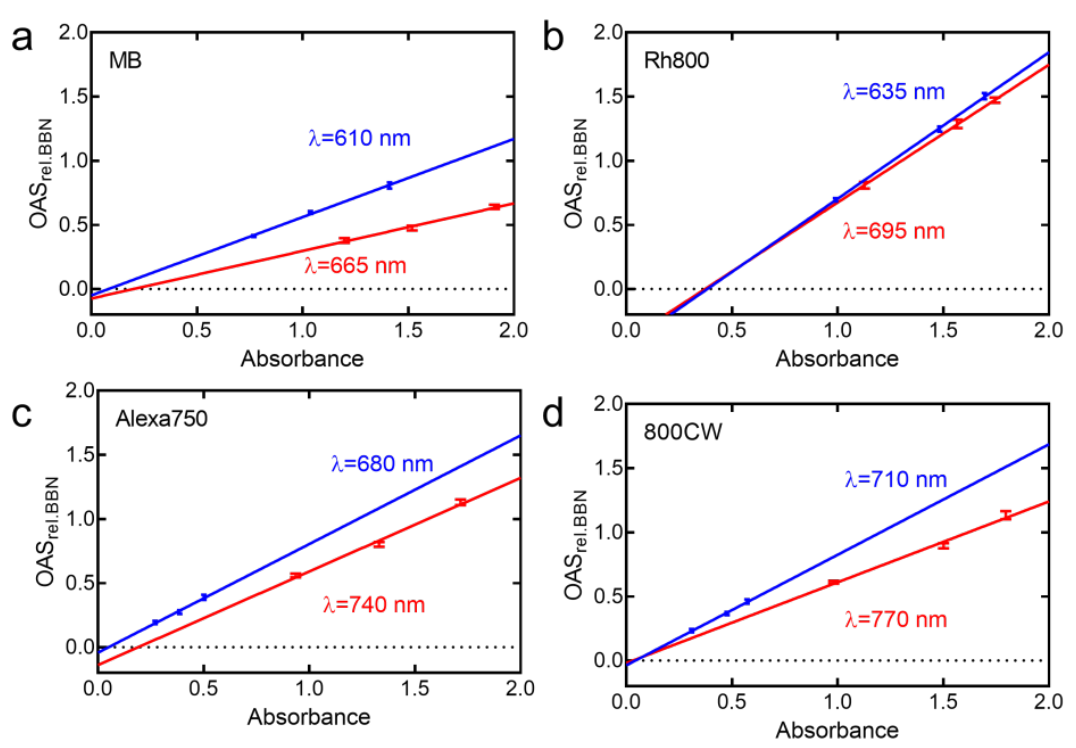

Supplementary Figure 6 linear relationship of OAS and absorbance for the main peaks and shoulders(a) MB - Methylene blue, (b) Rh800 - Rhodamine 800, (c) Alexa750 - Alexa Fluor 750 and (d) $800 \mathrm{CW}$ - IRDye $800 \mathrm{CW}$. 


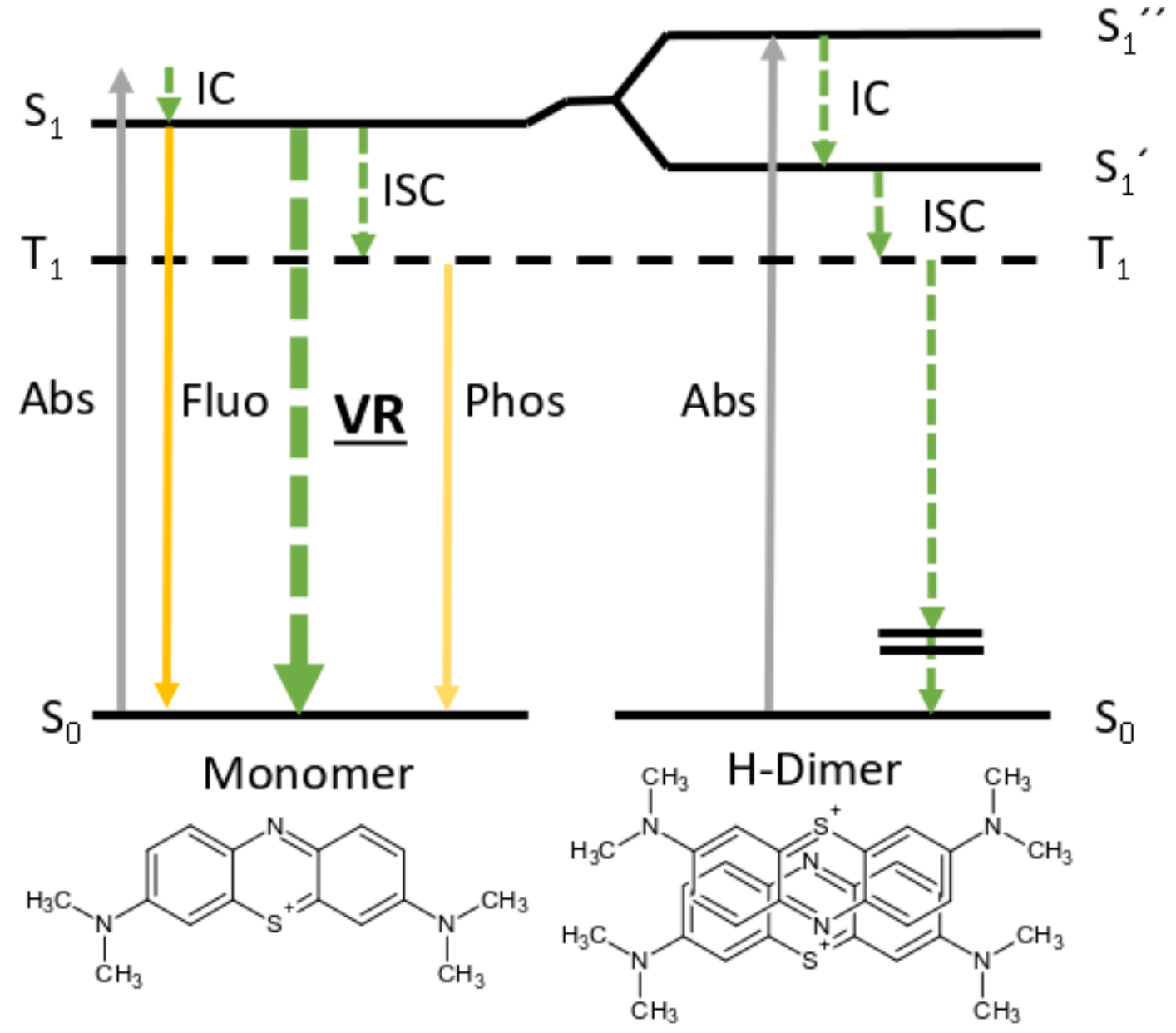

Supplementary Figure 7 Jablonski energy diagram for monomers and geometrically perfect $\mathrm{H}$-type dimers of Methylene blue. Plain lines represent radiative transitions, dashed lines represent nonradiative processes. Abs: absorbance, $\mathrm{S}_{0}$ : ground state, $\mathrm{S}_{1}$ : singlet excited state, $\mathrm{T}_{1}$ : primary triplet excited state, $S^{\prime}{ }_{1}$ and $S^{\prime \prime}{ }_{1}$ : split exciton states, VR: vibrational relaxation, ISC: intersystem crossing, IC: internal conversion, Pho: phosphorescence, Fluo: fluoresence. The double bar in the relaxation from $\mathrm{T}_{1}$ indicates a chance for discontinuity in the transition diagram: the dimer triplet can undergo electron transfer. Bottom: possible configurations of MB monomer and H-type dimer. Modify from Kasha et al. 196526 and Morgounova et al. $2013^{27}$. 

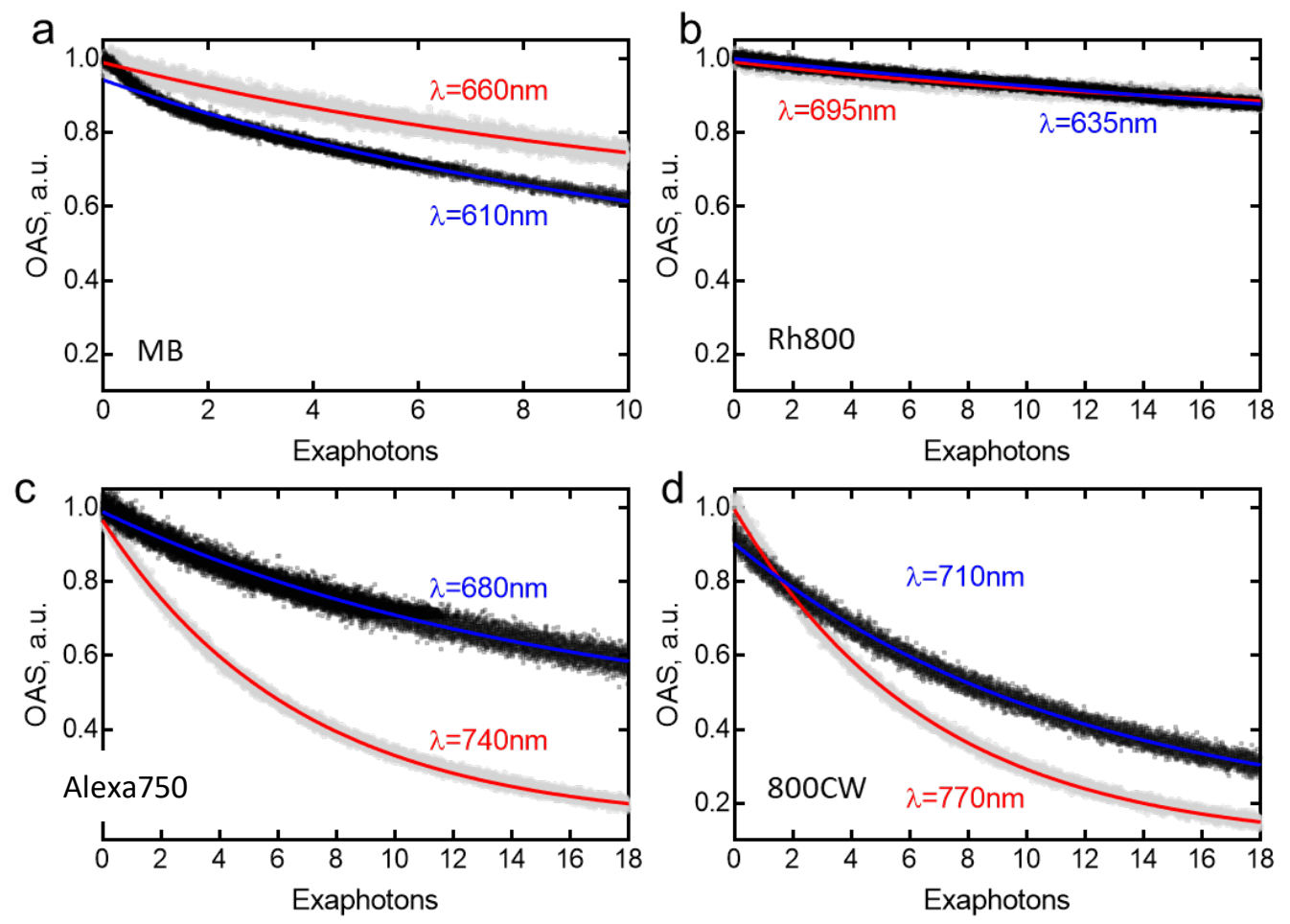

Supplementary Figure 8 Bleaching measured as optoacoustic signal of (a) Methylene Bue - MB, (b) Rhodamine 800 - Rh800, (c) Alexa Fluor 750 - Alexa750, and (d) IRDye 800CW at shoulder and main peak wavelengths. 


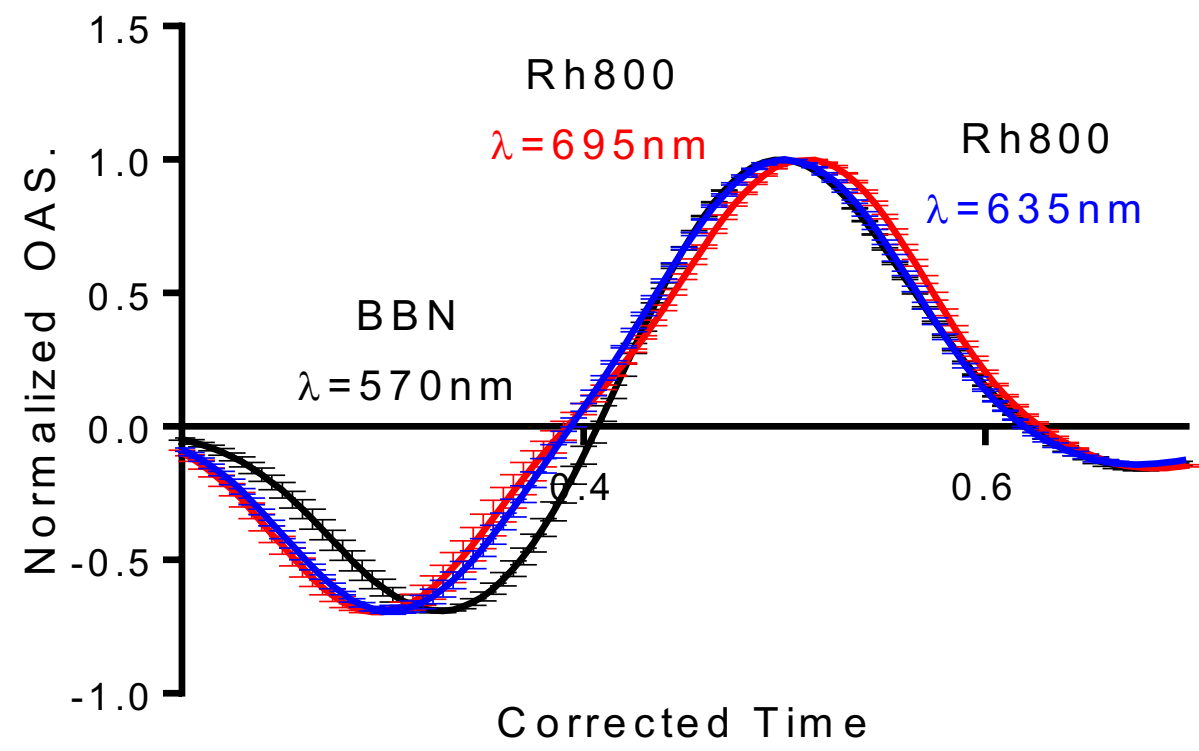

Supplementary Figure 9 Optoacoustic signal for Rh800 at $695 \mathrm{~nm}$ and $635 \mathrm{~nm}$; and BBN at $570 \mathrm{~nm}$. 

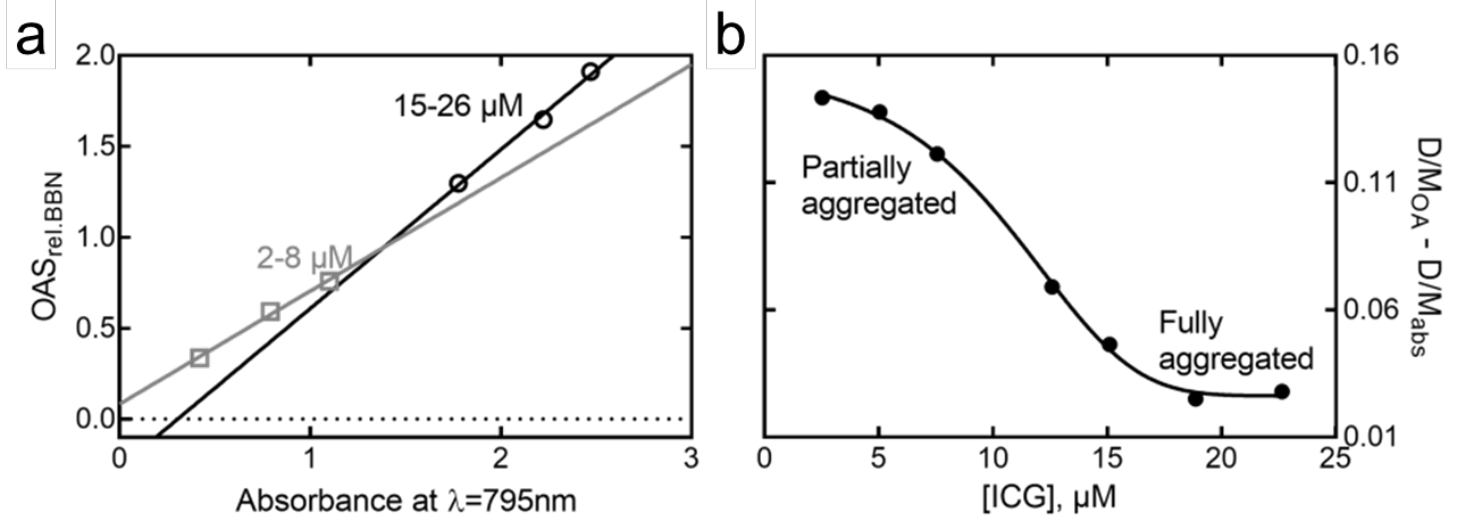

Supplementary Figure 10 (a) Segmented linear relation between absorbance and optoacoustic signal for ICG. (b) Difference between the dimer/monomer ratio from absorbance and OA at increasing ICG concentration. 

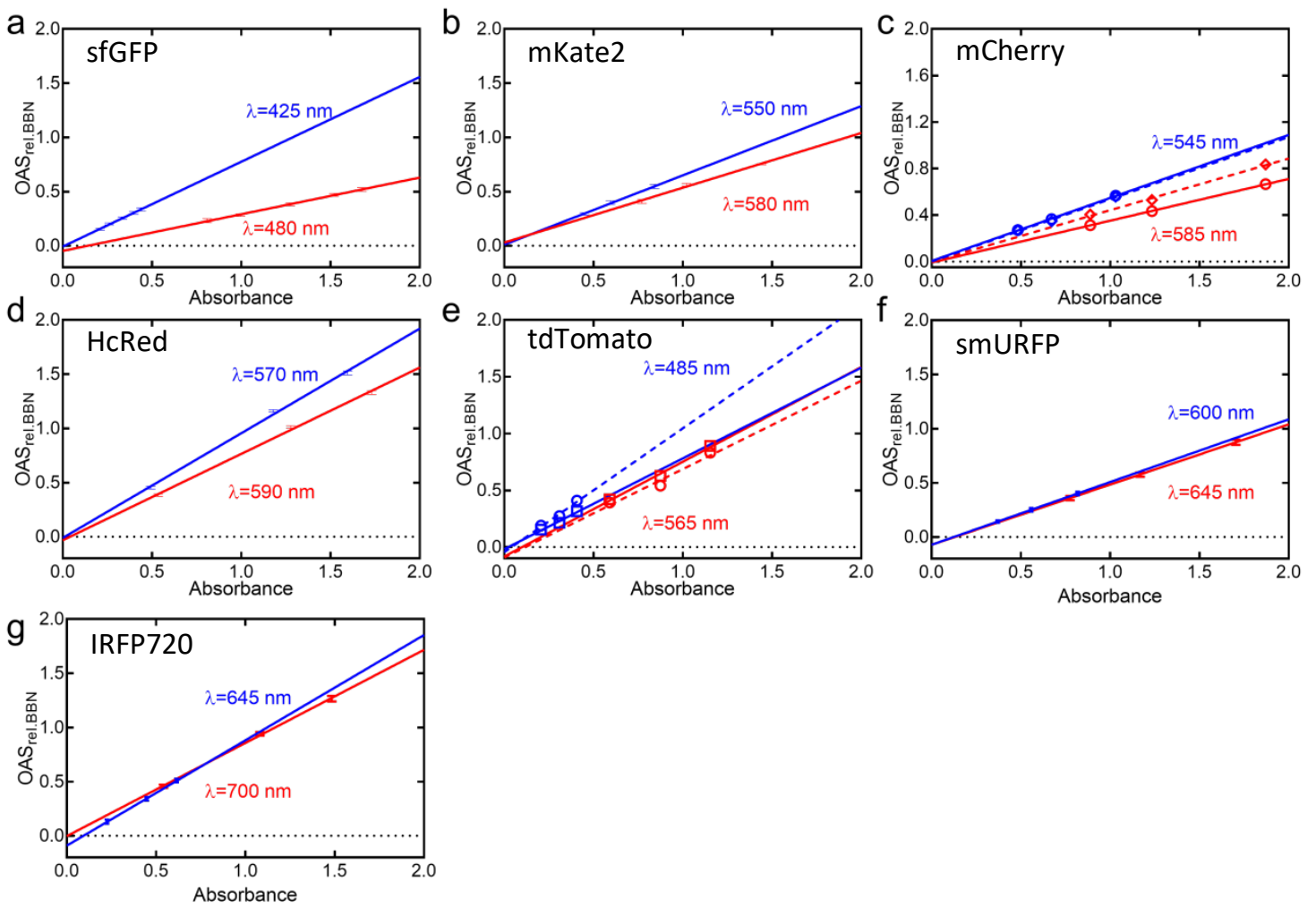

Supplementary Figure 11 Linear relationship of OAS and absorbance for the main peaks and shoulders for (a) sfGFP, (b) mKate2, (c) mCherry, (d) HcRed, (e) tdTomato, (f) smURFP, and (g) IRFP720. 


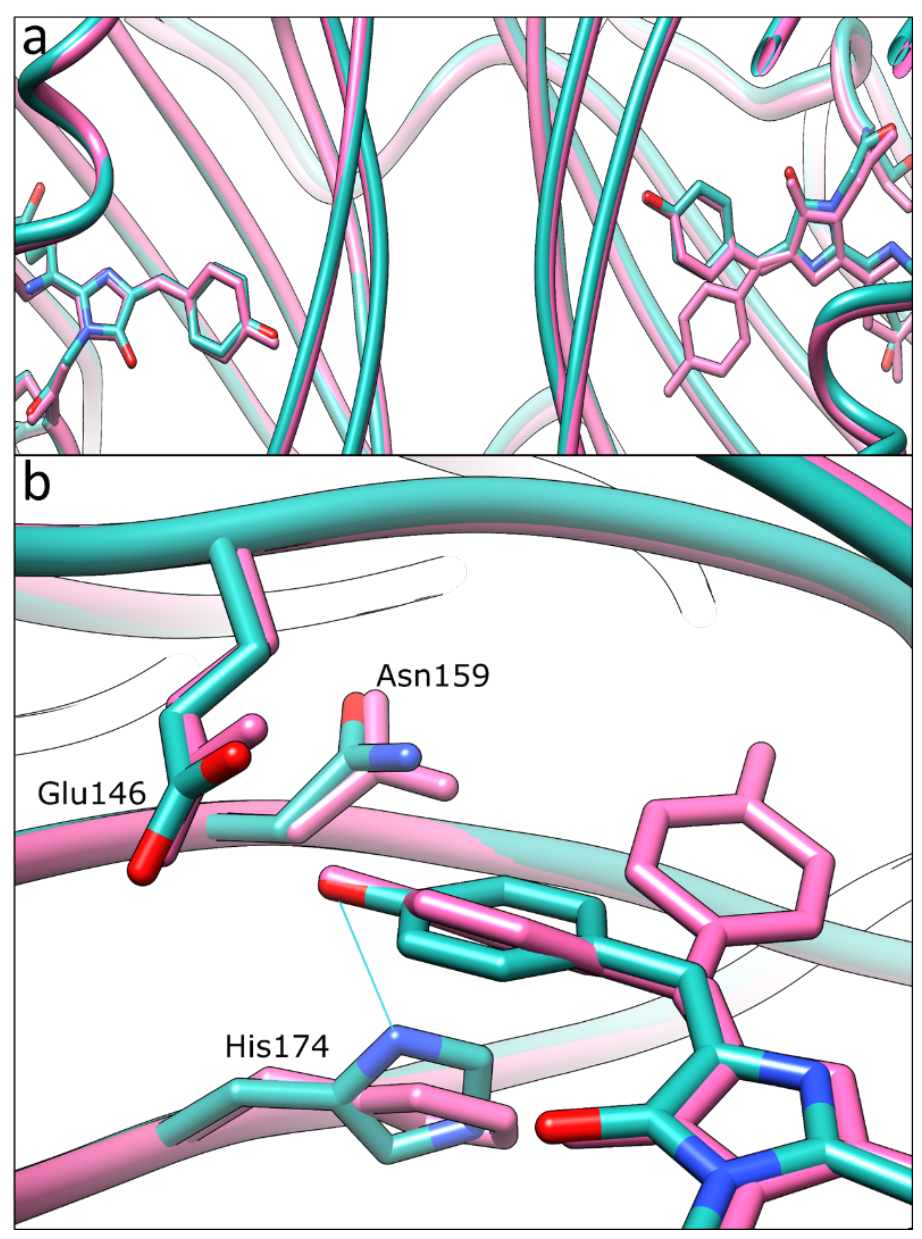

Supplementary Figure 12 In blue structure of HcRed after 60000 pulses of $565 \mathrm{~nm}$ light and in pink structure of native HcRed (PDB code 1YZW): (a) dimer interface, and (b) Hydrogen bond between His174 and trans-nonplanar conformer in illuminated HcRed (blue). 
Suppl. Table 2. Data collection and refinement statistics for HcRed .

\begin{tabular}{|c|c|}
\hline Data collection & \\
\hline PDB ID & $6 Y 1 G$ \\
\hline Beamline & SLS PXIII X06DA \\
\hline Wavelength & 0.999995 \\
\hline Space group & $P 2_{1} 2_{1} 2_{1}$ \\
\hline Cell dimensions $\left(\AA{ }^{\circ},{ }^{\circ}\right)$ & $\begin{array}{c}50.7175 .20 \\
254.21\end{array}$ \\
\hline $\begin{array}{l}\text { No. of molecules per } \\
\text { asymmetric unit }\end{array}$ & 4 \\
\hline Resolution $(\AA)$ & $\begin{array}{c}50-2.3 \\
(2.36-2.30)\end{array}$ \\
\hline$R_{\text {merge }}$ & 27.9 \\
\hline$I / \sigma l$ & $9.9(1.7)$ \\
\hline $\mathrm{CC}(1 / 2)$ & $99.5(67.3)$ \\
\hline Completeness (\%) & $99.7(99.4)$ \\
\hline Redundancy & $13.3(13.3)$ \\
\hline \multicolumn{2}{|l|}{ Refinement } \\
\hline Resolution (Å) & 2.30 \\
\hline No. reflections & 44,173 \\
\hline$R_{\text {work }} / R_{\text {free }}$ & $16.8 / 24.7$ \\
\hline \multicolumn{2}{|l|}{ No. atoms } \\
\hline Protein & 7,167 \\
\hline Water & 596 \\
\hline Other & 76 \\
\hline$B$-factor overall & 30.0 \\
\hline \multicolumn{2}{|l|}{ R.m.s. deviations } \\
\hline Bond lengths $(\AA ̊)$ & 0.012 \\
\hline Bond angles $\left({ }^{\circ}\right)$ & 1.965 \\
\hline \multicolumn{2}{|l|}{ Ramachandran plot } \\
\hline Most favored (\%) & 97 \\
\hline Additional allowed (\%) & 3 \\
\hline
\end{tabular}

*Values in parentheses are for highest-resolution shell. 
Aggregation state of ICG
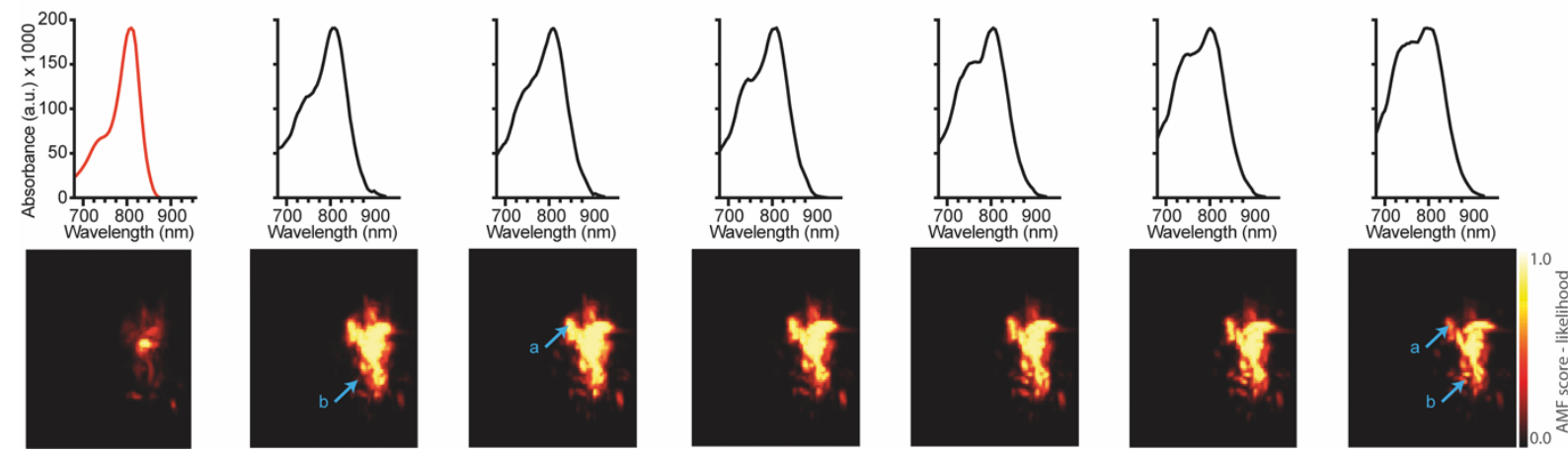

Supplementary Figure 13 Adaptive matched filtering (AMF) spectral unmixing of a colon slice of an euthanized mouse filled rectally with solution of ICG. Different aggregation states are present in different regions - presumably indicating different concentrations or conditions of ICG in the different areas of the colon. 


\section{References for Supplementary text:}

(1) Deán-Ben, X. L.; Gottschalk, S.; Mc Larney, B.; Shoham, S.; Razansky, D. Advanced Optoacoustic Methods for Multiscale Imaging of: In Vivo Dynamics. Chem. Soc. Rev. 2017, 46 (8), 2158-2198. https://doi.org/10.1039/c6cs00765a.

(2) Land, E. J. THE TRIPLET EXCITED STATES OF BILIVERDIN AND BILIVERDIN DIMETHYL ESTER. Photochem. Photobiol. 1979, 29 (3), 483-487. https://doi.org/10.1111/j.17511097.1979.tb07079.x.

(3) Schaberle, F. A.; Rego Filho, F. de A. M. G.; Reis, L. A.; Arnaut, L. G. Assessment of Lifetime Resolution Limits in Time-Resolved Photoacoustic Calorimetry vs. Transducer Frequencies: Setting the Stage for Picosecond Resolution. Photochem. Photobiol. Sci. 2016, 15 (2), 204210. https://doi.org/10.1039/c5pp00397k.

(4) S. P. McGlynn, T. A. and M. K. Molecular Spectroscopy of the Triplet State; Prentice-Hall: New Jersey, 1969.

(5) Mishra, K.; Fuenzalida-Werner, J. P. J. P.; Ntziachristos, V.; Stiel, A. C. Photocontrollable Proteins for Optoacoustic Imaging. Anal. Chem. 2019, 91 (9).

https://doi.org/10.1021/acs.analchem.9b01048.

(6) Rosencwaig, A. Photoacoustic Spectroscopy. Annu. Rev. Biophys. Bioeng. 1980, 9 (1), 31-54. https://doi.org/10.1146/annurev.bb.09.060180.000335.

(7) Braslavsky, S. E.; Ellul, R. M.; Weiss, R. G.; Al-Ekabi, H.; Schaffner, K.; Alekabi, H.; Schaffner, K.; Al-Ekabi, H.; Schaffner, K.; Silvia E. Braslavsky Richard G. Weiss, Hussain Al-Ekabi, Kurt Schaffner, R. M. E. Photoprocesses in Biliverdin Dimethyl Ester in Ethanol Studied by LaserInduced Optoacoustic Spectroscopy (Lioas). Tetrahedron 1983, 39 (11), 1909-1913. https://doi.org/https://doi.org/10.1016/S0040-4020(01)88704-4.

(8) Laufer, J.; Jathoul, A.; Pule, M.; Beard, P. In Vitro Characterization of Genetically Expressed Absorbing Proteins Using Photoacoustic Spectroscopy. Biomed. Opt. Express 2013, 4 (11), 2477. https://doi.org/10.1364/BOE.4.002477.

(9) Brown, J. E.; Diaz, L.; Christoff-Tempesta, T.; Nesbitt, K. M.; Reed-Betts, J.; Sanchez, J.; Davies, K. W. Characterization of Nitrazine Yellow as a Photoacoustically Active PH Reporter Molecule. Anal. Chem. 2015, 87 (7), 3623-3630. https://doi.org/10.1021/ac503515k.

(10) Abbruzzetti, S.; Viappiani, C.; Murgida, D. H.; Erra-Balsells, R.; Bilmes, G. M. Non-Toxic, Water-Soluble Photocalorimetric Reference Compounds for UV and Visible Excitation. Chem. Phys. Lett. 1999, 304 (3-4), 167-172. https://doi.org/10.1016/S0009-2614(99)00306-1.

(11) Rios, A. de O.; Mercadante, A. Z.; Borsarelli, C. D. Triplet State Energy of the Carotenoid Bixin Determined by Photoacoustic Calorimetry. Dye. Pigment. 2007, 74 (3), 561-565.

https://doi.org/10.1016/j.dyepig.2006.03.018.

(12) Borges Dos Santos, R. M.; Lagoa, A. L. C.; Martinho Simões, J. A. Photoacoustic Calorimetry. An Examination of a Non-Classical Thermochemistry Tool. J. Chem. Thermodyn. 1999. https://doi.org/10.1006/jcht.1999.0513.

(13) Vetschera, P.; Mishra, K.; Fuenzalida-Werner, J. P.; Chmyrov, A.; Ntziachristos, V.; Stiel, A. C. Characterization of Reversibly Switchable Fluorescent Proteins in Optoacoustic Imaging. Anal. Chem. 2018, 90 (17), 10527-10535. https://doi.org/10.1021/acs.analchem.8b02599.

(14) Beard, P. Biomedical Photoacoustic Imaging. Interface Focus 2011, 1 (4), 602-631. https://doi.org/10.1098/rsfs.2011.0028.

(15) Cox, B.; Laufer, J. G.; Arridge, S. R.; Beard, P. C. Quantitative Spectroscopic Photoacoustic Imaging: A Review. J. Biomed. Opt. 2012, 17 (6), 61202. https://doi.org/10.1117/1.JBO.17.6.061202.

(16) Tzoumas, S.; Nunes, A.; Olefir, I.; Stangl, S.; Symvoulidis, P.; Glasl, S.; Bayer, C.; Multhoff, G.; Ntziachristos, V. Eigenspectra Optoacoustic Tomography Achieves Quantitative Blood Oxygenation Imaging Deep in Tissues. Nat. Commun. 2016, 7 (May), 12121. https://doi.org/10.1038/ncomms12121.

(17) Laufer, J.; Zhang, E.; Beard, P. Evaluation of Absorbing Chromophores Used in Tissue Phantoms for Quantitative Photoacoustic Spectroscopy and Imaging. IEEE J. Sel. Top. Quantum Electron. 2010, 16 (3), 600-607. https://doi.org/10.1109/JSTQE.2009.2032513.

(18) Kabsch, W. Evaluation of Single-Crystal X-Ray Diffraction Data from a Position-Sensitive Detector. J. Appl. Crystallogr. 1988, 21 (6), 916-924. https://doi.org/10.1107/S0021889888007903.

(19) Evans, P. Scaling and Assessment of Data Quality. Acta Crystallogr. Sect. D Biol. Crystallogr. 2006, 62 (1), 72-82. https://doi.org/10.1107/S0907444905036693.

(20) Winn, M. D.; Ballard, C. C.; Cowtan, K. D.; Dodson, E. J.; Emsley, P.; Evans, P. R.; Keegan, R. M.; Krissinel, E. B.; Leslie, A. G. W.; McCoy, A.; et al. Overview of the CCP 4 Suite and Current Developments. Acta Crystallogr. Sect. D Biol. Crystallogr. 2011, 67 (4), 235-242. 
https://doi.org/10.1107/S0907444910045749.

(21) French, S.; Wilson, K. On the Treatment of Negative Intensity Observations. Acta Crystallogr. Sect. A 1978. https://doi.org/10.1107/S0567739478001114.

(22) Collaborative Computational Project, N. 4. The CCP4 Suite: Programs for Protein Crystallography. Acta Crystallogr. Sect. D Biol. Crystallogr. 1994, 50 (5), 760-763. https://doi.org/10.1107/S0907444994003112.

(23) Wilmann, P. G.; Petersen, J.; Pettikiriarachchi, A.; Buckle, A. M.; Smith, S. C.; Olsen, S.; Perugini, M. a.; Devenish, R. J.; Prescott, M.; Rossjohn, J. The 2.1 A Crystal Structure of the Far-Red Fluorescent Protein HcRed: Inherent Conformational Flexibility of the Chromophore. J. Mol. Biol. 2005, 349, 223-237. https://doi.org/10.1016/j.jmb.2005.03.020.

(24) Laskowski, R. A.; MacArthur, M. W.; Moss, D. S.; Thornton, J. M. PROCHECK: A Program to Check the Stereochemical Quality of Protein Structures. J. Appl. Crystallogr. 1993. https://doi.org/10.1107/s0021889892009944.

(25) Chen, V. B.; Arendall, W. B.; Headd, J. J.; Keedy, D. A.; Immormino, R. M.; Kapral, G. J.; Murray, L. W.; Richardson, J. S.; Richardson, D. C. MolProbity: All-Atom Structure Validation for Macromolecular Crystallography. Acta Crystallogr. Sect. D Biol. Crystallogr. 2010. https://doi.org/10.1107/S0907444909042073.

(26) Kasha, M.; Rawls, H. R.; El-Bayoumi, M. A. The Exciton Model In Molecular Spectroscopy. Pure Appl. Chem. 1965, 11 (3-4), 371-392. https://doi.org/10.1351/pac196511030371.

(27) Morgounova, E.; Shao, Q.; Hackel, B. J.; Thomas, D. D.; Ashkenazi, S. Photoacoustic Lifetime Contrast between Methylene Blue Monomers and Self-Quenched Dimers as a Model for DualLabeled Activatable Probes. J. Biomed. Opt. 2013, 18 (5), 56004. https://doi.org/10.1117/1.jbo.18.5.056004. 